\title{
Globale Sicht des Klimawandels
}

Hauke Schmidt, Veronika Eyring, Mojib Latif, Diana Rechid, Robert Sausen

2.1 Geschichte der Klimamodellierung - 8

2.2 Komponenten des Klimasystems, Prozesse und Rückkopplungen - 8

2.3 Ensembles von Klimamodellen und Szenarien - 10

2.3.1 Beschreibung der Szenarien - 11

2.4 IPCC-Bericht: Fortschritte und Schlüsselergebnisse - 11

2.4.1 Simulation des historischen Klimawandels - 11

2.4.2 Projektionen des zukünftigen Klimas - 13

2.5 Kurz gesagt -15

Literatur - 15 
Eine Vielzahl von Beobachtungen zeigt, dass sich das Klima ändert. Um der Gesellschaft eine informierte Antwort darauf zu ermöglichen, ist es notwendig, Natur und Ursachen des Wandels $\mathrm{zu}$ verstehen und die mögliche zukünftige Entwicklung zu charakterisieren. In der Klimaforschung sind numerische Modelle dafür unverzichtbare Werkzeuge. Sie beruhen auf mathematischen Gleichungen, die das Klimasystem oder Teile davon abbilden und sich nur mithilfe von Computern berechnen lassen. Die Klimamodelle helfen uns, das komplexe Zusammenspiel verschiedener Komponenten und Prozesse im Erdsystem zu verstehen und Beobachtungen zu interpretieren. Mit Modellen lassen sich Projektionen des künftigen Klimas erstellen. Diese liefern Antworten auf die Frage: „Was wäre, wenn?“ Wie entwickelt sich das Klima unter bestimmten Bedingungen, beispielsweise wenn der Mensch zusätzliche Treibhausgase in die Atmosphäre entlässt? Oder: Welchen Effekt hätte ein großer Vulkanausbruch auf das Klima?

\subsection{Geschichte der Klimamodellierung}

Die aktuell verwendeten Klimamodelle sind das Ergebnis einer seit über einem halben Jahrhundert andauernden und bei weitem nicht abgeschlossenen Entwicklung. Das erste Modell, das auf physikalischen Grundlagen beruht, war ein eindimensionales Strahlungskonvektionsmodell (Manabe und Möller 1961). Darin sorgen Sonneneinstrahlung und vertikale Luftströmungen für eine stabile vertikale Temperaturverteilung auf der Erde - es stellt sich eine Gleichgewichtstemperatur ein. Seit 1969 rechnen Energiebilanzmodelle mit der Energie von Strahlungs- und Wärmeflüssen (Budyko 1969; Sellers 1969). Obwohl die einfachsten dieser Modelle den horizontalen Wärmetransport vernachlässigen, lässt sich mit ihnen abschätzen, wie empfindlich die Gleichgewichtstemperatur an der Erdoberfläche etwa gegenüber Änderungen der Sonneneinstrahlung reagiert. Heute werden dreidimensionale atmosphärische Zirkulationsmodelle (atmospheric general circulation models, AGCMs) verwendet. Diese stammen aus der Wettervorhersage: Der Meteorologe Norman Phillips fragte sich 1956, ob die Modelle zur Wettervorhersage auch die allgemeine Zirkulation der Atmosphäre und damit das Klima wiedergeben würden (Phillips 1956). Obwohl er in seinem Experiment nicht mehr als 30 Tage simulieren konnte, wird es häufig als die erste Klimasimulation angesehen. Die moderne Klimamodellierung und Wettervorhersage basieren auch weiterhin auf der rechnerischen Lösung ähnlicher Gleichungssysteme.

Bahnbrechend war die Simulation der Klimaeffekte, die aus einer Verdopplung des Kohlendioxidgehalts in der Atmosphäre resultieren (Manabe und Wetherald 1967). Das Modell verwendete eine idealisierte Verteilung von Land und Meer und vernachlässigte den täglichen und saisonalen Zyklus der Sonneneinstrahlung. Dennoch zeigte die Berechnung erstmals das Temperaturmuster der Erde mit einem starken Land-SeeKontrast und einer maximalen Erwärmung in den hohen nördlichen Breiten. Die „Mutter“ der heutigen Klimamodelle, das erste gekoppelte Atmosphäre-Ozean-Zirkulationsmodell, entstand 1969 (Manabe und Bryan 1969). Inzwischen werden vermehrt sogenannte Erdsystemmodelle (ESM) verwendet, die außer den
Komponenten Atmosphäre, Landoberfläche, Ozean und Meereis auch den Kohlenstoffkreislauf und andere interaktive Komponenten wie Aerosole (atmosphärische Mikropartikel, die die Strahlungsbilanz beeinflussen und eine Lufttrübung bewirken) berücksichtigen. In einem solchen Modell kann z. B. berücksichtigt werden, dass ein wärmerer Ozean tendenziell weniger $\mathrm{CO}_{2}$ aufnimmt, sodass mehr $\mathrm{CO}_{2}$ in der Atmosphäre bleibt.

Es handelt sich dabei also um einen positiven, d. h. einen die Reaktion des Klimas auf menschengemachte Antriebe verstärkenden Rückkopplungseffekt. Der schwedische Physiker und Chemiker Svante August Arrhenius untersuchte 1896 als Erster die Änderung der Oberflächentemperatur in Abhängigkeit von der $\mathrm{CO}_{2}$-Konzentration (Arrhenius 1896). Er berechnete eine Gleichgewichtsklimasensitivität von etwa $6^{\circ} \mathrm{C}$, spekulierte aber, dass sie möglicherweise überschätzt klein sein könnte. Die Gleichgewichtsklimasensitivität gibt an, wie sich die globale Erdoberflächentemperatur langfristig ändern würde, wenn sich die $\mathrm{CO}_{2}$-Konzentration verdoppelte ( Abschn. 2.2). Auf der Basis von nur zwei Klimamodellen schätzte die US-amerikanische $\mathrm{Na}$ tional Academy of Sciences 1979 einen Wert zwischen 1,5 und $4,5^{\circ} \mathrm{C}$ (Charney et al. 1979). In den vergangenen Jahrzehnten haben sich die Schätzungen kaum verändert. Auch im jüngsten, dem Fünften Sachstandsbericht des Weltklimarats (IPCC 2013a und 2013b), findet man diese Temperaturspanne.

Die sogenannte transiente Klimasensitivität gibt an, um wie viel Grad Celsius die globale Erdoberflächentemperatur zum Zeitpunkt der $\mathrm{CO}_{2}$-Verdopplung angestiegen ist: Berechnungen zufolge hat sich die Erde dann mit einer Wahrscheinlichkeit von $90 \%$ bereits um 0,9 bis $2,0^{\circ} \mathrm{C}$ erwärmt (Otto et al. 2013). Selbst wenn die $\mathrm{CO}_{2}$-Konzentration danach nicht mehr steigen sollte, würden sich die Troposphäre, d.h. die Atmosphäre bis in etwa $10 \mathrm{~km}$ Höhe, und die Ozeane weiter erwärmen - so lange, bis eine Gleichgewichtstemperatur erreicht ist.

Weltweit sind sich die Klimaforscher weitgehend einig: Nach dem jetzigen Kenntnisstand wird sich die Erde weiter erwärmen, wenn noch mehr Treibhausgase die Atmosphäre belasten. Wie sehr sich die Erde tatsächlich erwärmt, wird von den zukünftigen Treibhausgasemissionen abhängen - das zeigen die Projektionen für verschiedene Zukunftsszenarien ( Abschn. 2.4.2). Außerdem ist zu erwarten, dass sich Regionen sehr unterschiedlich stark erwärmen.

\subsection{Komponenten des Klimasystems, Prozesse und Rückkopplungen}

Die wesentlichen Komponenten des Klimasystems sind:

- die Atmosphäre,

- der Ozean mit seinem Meereis und seiner Biosphäre,

- die Landoberfläche mit der Landbiosphäre sowie den oberund unterirdischen Wasserflüssen und

- die Eisschilde inklusive der Schelfeise.

Das Wettergeschehen spielt sich in der Troposphäre ab. Wichtige Kenngrößen des Wetters sind u. a. Druck, Temperatur, Wind und die Komponenten des Wasserkreislaufs wie Wasserdampfgehalt, Niederschlag und Bewölkung. Über diese Größen erfährt der 


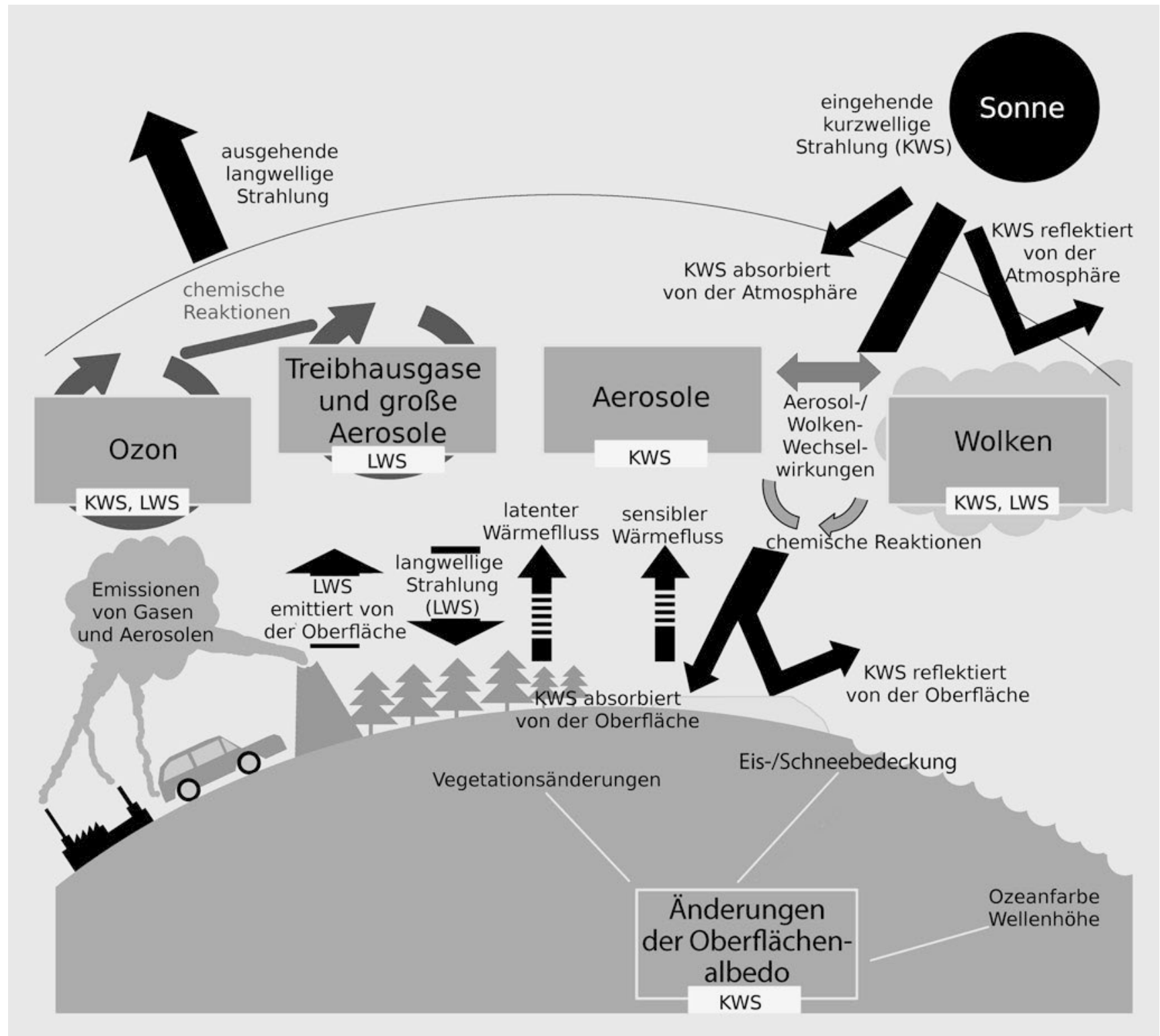

- Abb. 2.1 Wesentliche Antriebe des Klimasystems: Globale Klimaantriebe stören das Strahlungsgleichgewicht zwischen einfallender kurzwelliger Strahlung (KWS) von der Sonne und in den Weltraum hinausgehender langwelliger Strahlung (LWS). Von Menschen verursachte Emissionen von Gasen und Aerosolen greifen in den Strahlungshaushalt ein: entweder direkt als Treibhausgase oder Aerosole oder indirekt über chemische Reaktionen wie die Änderung der Ozonkonzentration über sekundär gebildete Aerosole oder Änderungen der Wolkeneigenschaften und Wolkenbedeckung. Außerdem verändert der Mensch die Oberflächeneigenschaften der Erde, besonders die Rückstreuung von KWS durch das Erdsystem. Zu den anthropogenen Antrieben kommen natürliche hinzu, z. B. Schwankungen der solaren Einstrahlung oder Emissionen durch Vulkane und natürliche Waldbrände. (Sausen nach IPCC)

Mensch das Wetter und seine langfristige Statistik - das Klima. Der entscheidende Antrieb des Klimasystems (- Abb. 2.1) ist die Sonneneinstrahlung, die vom Ort sowie von der Tages- und Jahreszeit abhängt. Sowohl die Erdoberfläche als auch die Wolken streuen einen Teil dieser (kurzwelligen) Strahlung direkt zurück in den Weltraum. Der größere Teil der Strahlung wird jedoch vom Boden, also von den Ozeanen und dem Land, sowie von Wolken und Spurenstoffen (Gase und Mikropartikeln) in die Atmosphäre aufgenommen und führt zu deren Erwärmung. Die so vom Klimasystem aufgenommene Strahlungsenergie der Sonne wird sowohl vom Boden als auch von strahlungsaktiven Substanzen in der Atmosphäre über (langwellige) Wärmestrahlung in den Weltraum geschickt. Dieses sind vor allem die
Treibhausgase (s.u.), aber auch feste und flüssige Partikel wie Wolkentropfen, Eiskristalle oder Aerosole. Langfristig besteht ein Gleichgewicht zwischen einfallender und ausgehender Strahlung. Da die Ausstrahlung des Klimasystems in den Weltraum zeitlich und räumlich wesentlich gleichmäßiger erfolgt als die Sonneneinstrahlung, gibt es einen Energiegewinn in den Tropen und einen Energieverlust in hohen Breiten. Wärmetransport durch Strömungen in der Atmosphäre und im Ozean gleicht diesen Unterschied aus.

Die langwellige Ausstrahlung gelangt zu einem großen Teil nicht direkt in den Weltraum, sondern wird von den Treibhausgasen, insbesondere Wasserdampf, Kohlendioxid, Methan, Distickstoffoxid (Lachgas) und Ozon, absorbiert und in alle Rich- 
tungen, also auch zum Erdboden hin, wieder emittiert. Dieser Treibhauseffekt sorgt dafür, dass in Bodennähe Temperaturen herrschen, die in den meisten Gebieten der Erde Leben ermöglichen.

Wasserdampf ist zwar für den größten Anteil am Treibhauseffekt verantwortlich, hat jedoch eine kurze Lebensdauer und reagiert schnell, insbesondere auf Temperaturveränderungen. Wenn es aufgrund einer Erhöhung der Konzentration anderer, zumeist langlebigerer und damit in der unteren Atmosphäre gut durchmischter Treibhausgase zu einem Temperaturanstieg in der Troposphäre kommt, zieht dies auch eine Erhöhung des Wasserdampfgehalts der Atmosphäre nach sich und verstärkt die Temperaturerhöhung - ein positiver Rückkopplungseffekt (z. B. Lacis et al. 2010).

Kohlendioxid, Methan, Distickstoffoxid und Ozon stammen zum Teil direkt aus natürlichen Quellen, gelangen durch menschliche Einflüsse in die Atmosphäre oder werden durch chemische Prozesse gebildet. Daher gehören auch die chemischen Kreisläufe mit ihren Quellen, Transporten, Senken und Prozessen zum Klimasystem, beispielsweise die Kreisläufe von Kohlenstoff, Stickstoff und Schwefel oder die Ozonchemie. Auch die Aerosole, ob fest oder flüssig, sind sowohl im Bereich der Sonneneinstrahlung als auch der Wärmestrahlung aktiv. Viele Aerosole dienen zudem als Kondensationskerne bei der Wolkenbildung und beeinflussen diese damit.

Der Mensch greift in das Klimasystem ein, indem er Spurenstoffe freisetzt und die Erdoberfläche durch Landnutzung verändert. Letzteres beeinflusst den Wasserkreislauf und die Rückstreuung der Sonneneinstrahlung. Insbesondere durch Nutzung fossiler Brennstoffe hat sich der atmosphärische Volumenanteil des Kohlendioxids von einem vorindustriellen Wert von ca. 280 ppm auf etwa 400 ppm im Jahr 2015 erhöht. Diese erhöhte Treibhausgaskonzentration verstärkt, wie oben erläutert, den Treibhauseffekt und führt zur Erderwärmung. Gelangen dagegen Schwefelverbindungen in die Atmosphäre, leidet zwar die Luftqualität und es entsteht saurer Regen, jedoch kommt es durch verstärkte Rückstreuung der Sonneneinstrahlung auch zu einer Abkühlung.

Betrachtet man die von Menschen verursachten (anthropogenen) Änderungen der Konzentrationen von Treibhausgasen und anderer strahlungsaktiver Spurenstoffe sowie die direkten Folgen daraus für das Klima, so stellt man fest: Die tatsächliche Klimaänderung ist größer, als man es aufgrund des geänderten Strahlungsantriebs dieser Gase erwarten würde. Das liegt an den positiven Rückkopplungen im Klimasystem wie der oben genannten Wasserdampf- und Eis-Albedo-Rückkopplung. Letztere beruht darauf, dass bei einer Erwärmung an der Erdoberfläche das Meereis teilweise abschmilzt. Eis reflektiert jedoch Sonneneinstrahlung besser als Ozeanwasser. Weniger Eis reflektiert demzufolge weniger Sonneneinstrahlung, und die Erde erwärmt sich zusätzlich. Andererseits strahlt jeder Gegenstand, also auch die Erde, mit steigender Temperatur mehr Wärme ab. Das dämpft die Erwärmung der Atmosphäre - eine negative Rückkopplung.

Die gesamte Wirkung aller Rückkopplungen im Klimasystem kann man über die oben angesprochenen Klimasensitivitäten erfassen. Sie lassen sich nicht direkt messen, sondern nur durch Kombination von Messungen, z.B. auch von Temperatur- und
Treibhausgaskonzentrationsänderungen auf paläontologischen Zeitskalen und numerischen Studien abschätzen. Die relativ hohe Unsicherheit dieser Abschätzungen ( Abschn. 2.1) ist eine der Ursachen für Unsicherheiten in den Projektionen des zukünftigen Klimas ( Abschn. 2.4.2).

Wie wird die Menschheit in Zukunft das Klima verändern? Diese Frage kann man nur mithilfe der Klimamodelle untersuchen. Hier steht man vor einem Dilemma: Einerseits möchte man das wirkliche Klimasystem möglichst genau mit all seinen Prozessen, Rückkopplungen und Wirkungen beschreiben, andererseits reichen weder die gegenwärtigen Computerleistungen, um das in beliebiger Genauigkeit zu tun, noch sind alle grundlegenden Prozesse im Klimasystem hinreichend verstanden, um sie in einem numerischen Modell exakt abzubilden.

Politik, Wirtschaft und Gesellschaft sind aber auf wissenschaftlich fundierte Erkenntnisse über den Klimawandel angewiesen, um Entscheidungen zu treffen. Deshalb wird eine Hierarchie von Klimamodellen angewendet: Am einen Ende der Hierarchie stehen Modelle größtmöglicher Komplexität und Auflösung, die die gegenwärtigen Computerleistungen ausschöpfen, am anderen Ende konzeptionelle Modelle, in denen man versucht, wesentliche Prozesse des Klimasystems herauszudestillieren, um so zu einem besseren Verständnis zu gelangen (z. B. Bony et al. 2013). Diese benötigen eine geringere Rechnerleistung und erlauben daher eine große Zahl von Experimenten.

Wenn man sich der Grenzen der Modelle bewusst ist, lassen sich damit nützliche Erkenntnisse zum Klimawandel gewinnen.

\subsection{Ensembles von Klimamodellen und Szenarien}

Die Modellergebnisse des jüngsten Weltklimaberichts beruhen vor allem auf Simulationen mit ca. 40 verschiedenen Erdsystemmodellen (IPCC 2013a). Diese Simulationen wurden im Rahmen des internationalen Modellvergleichsprojekts Coupled Model Intercomparison Project Phase 5 (CMIP5) durchgeführt (Taylor et al. 2012). Ein Ziel des Projekts ist es, vergangene und mögliche künftige Klimaänderungen aufgrund anthropogener und natürlicher Strahlungsantriebe mithilfe mehrerer Modelle zu verstehen. Dazu werden regelmäßig die Randbedingungen der Simulationen neu bestimmt. Die Projektteilnehmer rechnen diese dann mit ihren Modellen und stellen die Ergebnisse in einem zentralen Datenarchiv für Analysen bereit. In CMIP5 unterscheidet man erstmals zwischen Langzeit- und dekadischen Simulationen. Letztere starten mit Beobachtungsdaten sich langsam ändernder Komponenten des Klimasystems wie Temperatur und Salzgehalt des Ozeans, da sie die aktuellen Schwankungen im Klimasystem widerspiegeln. Dekadische Simulationen sollen Aussagen für Jahre bis Jahrzehnte liefern - ein aktuelles Forschungsfeld.

Im Verlauf des CMIP wurden die Prozesse und Rückkopplungen in den Modellen erweitert und verbessert. Vor allem simulieren erstmals in CMIP5 viele Modelle den Kohlenstoffkreislauf interaktiv (Friedlingstein et al. 2014). Einige Modelle berücksichtigen chemische Prozesse (Eyring et al. 2013; Lamarque et al. 2013) sowie Aerosole (Flato et al. 2013). Ein wichtiger Fortschritt 


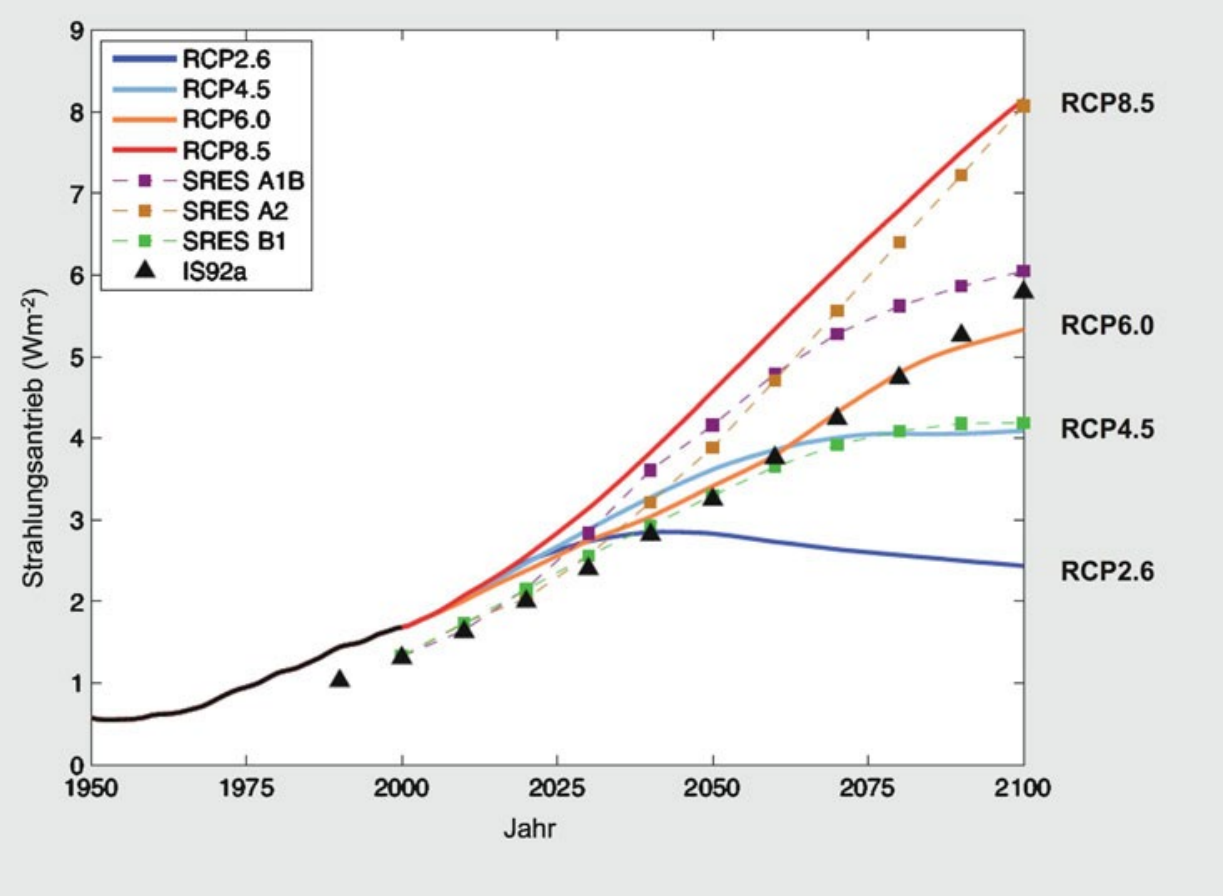

- Abb. 2.2 Anthropogen bedingte Veränderung des Strahlungsantriebs an der Tropopause (gemessen in Kohlenstoffäquivalenten) im Vergleich zum vorindustriellen Wert um 1765 für den historischen Zeitraum im 20. Jahrhundert und projiziert für das 21. Jahrhundert auf Basis der SRES-Emissionsszenarien (grau) (verwendet in CMIP3/AR4 2007) im Vergleich zu den RCPs (CMIP5/AR5 2013) und dem Szenario IS92a (AR2 1996). Die gesamte Veränderung der Strahlungsbilanz als Grundlage für die Klimaprojektionen ergibt sich aus anthropogen plus natürlich bedingtem Strahlungsantrieb. (IPCC 2013b, Abb. 1.15, S. 146)

ist auch die größere Anzahl von Simulationen mit den einzelnen Modellen und die größere Anzahl verwendeter Modelle, also ein größeres Modellensemble. Verschiedene Modelle reagieren auf einen gleichen Strahlungsantrieb unterschiedlich. Die dadurch hervorgerufene Schwankungsbreite der Ergebnisse wird häufig im Hinblick auf die Unsicherheit künftiger Klimaänderungen interpretiert. Dabei ist jedoch Vorsicht geboten, da etwaige systematische Fehler aller Modelle nicht ausgeschlossen werden können. Ebenfalls ist umstritten, ob Modelle, die Beobachtungen besser reproduzieren als andere, in einem Modellensemble stärkeres Gewicht erlangen sollen. Für die Meereisprojektionen wurde im Fünften Sachstandsbericht erstmalig nicht nur ein Mittelwert für das gesamte Modellensemble präsentiert, sondern auch für eine Auswahl von Modellen, die die beobachtete Meereisentwicklung der Vergangenheit am besten wiedergeben (IPCC 2013a).

\subsubsection{Beschreibung der Szenarien}

Während die Klimaprojektionen im Vierten Sachstandsbericht des IPCC auf den SRES-Emissionsszenarien beruhten (IPCC 2007; Nakicenovic und Swart 2000), verwendete man im Fünften IPCC-Bericht die sogenannten repräsentativen Konzentrationspfade (RCPs) (van Vuuren et al. 2011; Meinshausen et al. 2011). Im Vergleich zu den SRES-Emissionsszenarien decken die RCPs eine weitere Spanne möglicher Treibhausgaskonzentrationen und damit Strahlungsantriebe ab (• Abb. 2.2). Während die drei RCPs mit höheren Strahlungsantrieben für die Emissionen von $\mathrm{CO}_{2}$ und Methan $\left(\mathrm{CH}_{4}\right)$ nicht ganz die Breite der SRES-Szenarien abdecken, erweitert das Szenario RCP2.6 die Bandbreite deutlich nach unten. Je nach Modell und Experiment gehen die Konzentrationen oder die Emissionen der RCPs in die Simulationen ein, deren Ergebnisse dann die Grundlage für Klimaprojektionen bilden.

\subsection{IPCC-Bericht: Fortschritte und Schlüsselergebnisse}

Im Fünften Sachstandsbericht (IPCC 2013a) behandelt der Bericht der ersten Arbeitsgruppe die physikalischen Grundlagen des Klimawandels und benutzt eine einheitliche Sprachregelung zur Angabe von Wahrscheinlichkeiten und Unsicherheiten. So gilt eine Aussage als „sehr wahrscheinlich“, wenn sie mit mehr als 90-prozentiger Sicherheit zutrifft. Im Folgenden sind derartige Angaben durch Anführungszeichen als Zitat aus dem Bericht gekennzeichnet.

\subsubsection{Simulation des historischen Klimawandels}

Ein wichtiges Element des CMIP5-Projekts, dessen Ergebnisse im IPCC-Bericht verwendet werden, ist die Simulation des Klimas von 1850 bis 2005. Gespeist wird diese Simulation mit Daten aus Beobachtungen, insbesondere der zeitlichen Entwicklung der Zusammensetzung der Atmosphäre und der Sonneneinstrah- 


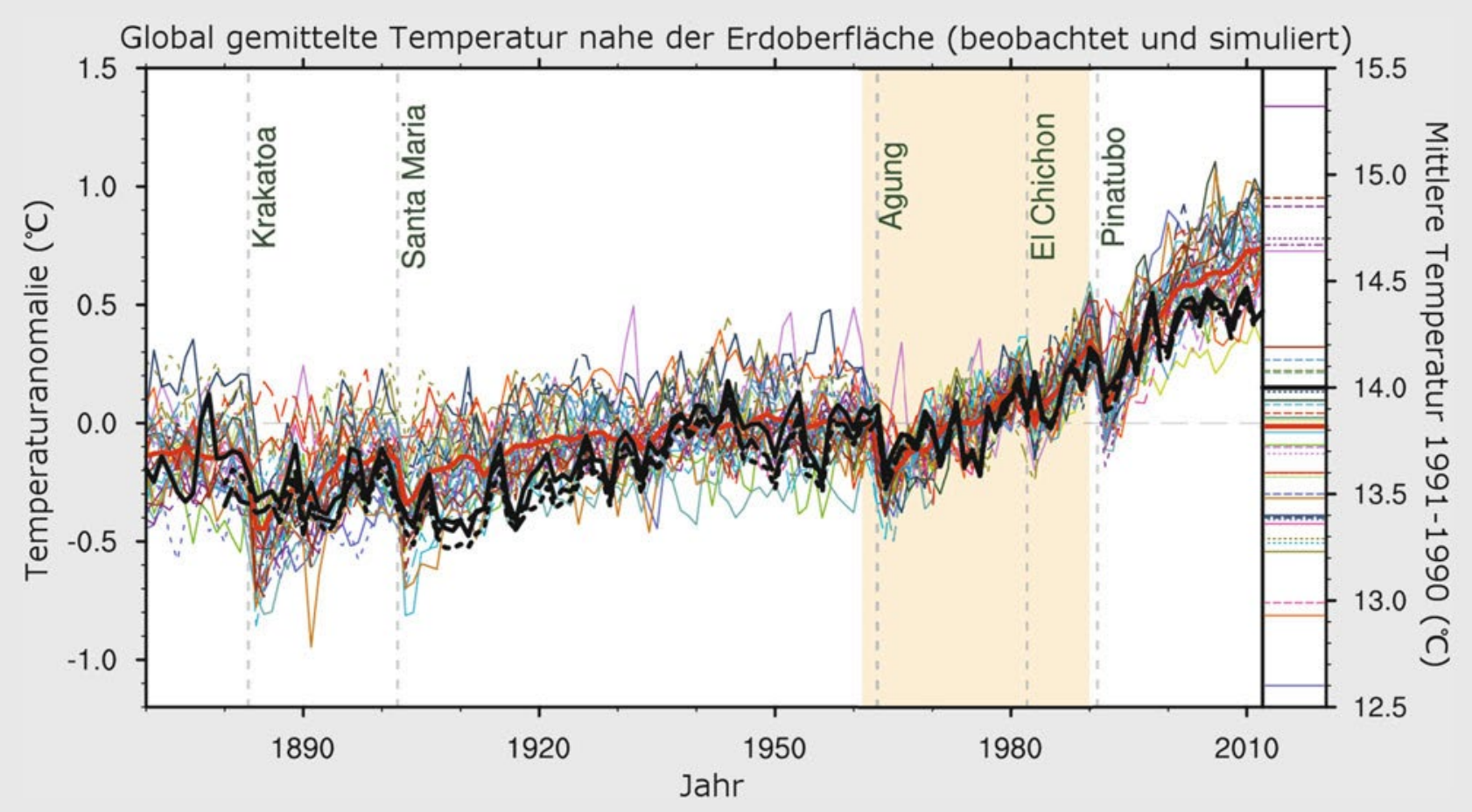

- Abb. 2.3 Beobachtete und simulierte global und jährlich gemittelte Oberflächentemperaturen, dargestellt als Abweichung von der mittleren Temperatur der Jahre 1961-1990 (gelb hinterlegt). Die vertikalen gestrichelten Linien markieren große Vulkanausbrüche. Gezeigt sind Einzelsimulationen der CMIP5-Modelle (dünne Linien), das Mittel über alle Modelle (dicke rote Linie) und drei verschiedene Beobachtungen (dicke schwarze Linien). Alle Simulationen nutzen historische Strahlungsantriebe bis 2005 und das RCP4.5-Szenario. Waagerechte Linien am rechten Rand: Mittelwerte der beobachteten und von den verschiedenen Modellen simulierten absoluten Oberflächentemperaturen der Jahre 1961-1990. (Modifizierte Abb. 9.8, IPCC-AR5-WG1, Flato et al. 2013)

lung. Ziel dieser Simulationen der Vergangenheit ist insbesondere die Bewertung der Modelle (Flato et al. 2013): Wenn ein Modell das beobachtete Klima annähernd widerspiegelt, steigt das Vertrauen in seine Prognosefähigkeit. Darüber hinaus liefern historische Simulationen Anfangszustände für die Projektionen des zukünftigen Klimas und dienen als Referenz.

Zur Bewertung wird die Klimavariabilität, die aus den Modellrechnungen hervorgeht, räumlich und zeitlich mit der beobachteten Variabilität verglichen. Verlässliche Beobachtungen oder Rekonstruktionen bis in die vorindustrielle Zeit zurück sind allerdings rar. Erst seit Beginn des Satellitenzeitalters hat sich die Beobachtungslage deutlich verbessert. Wie realistisch können die heutigen Modelle also langfristige Entwicklungen, aber auch saisonale Klimaschwankungen und Schwankungen zwischen einzelnen Jahren darstellen? Im Vergleich zu ihren Vorgängern können die Modelle von CMIP5 die Entwicklung bestimmter Kenngrößen besser abbilden, etwa der regionalen Oberflächentemperaturen, kontinentaler Niederschlagsmuster oder von Extremereignissen. Auch das El-Niño-Phänomen im tropischen Pazifik und den Rückgang des Meereises im arktischen Sommer reproduzieren diverse Modelle relativ realistisch selbst, wenngleich Letzterer im Mittel immer noch unterschätzt wird. Bei den regionalen Temperatur- und Niederschlagsmustern ist der in CMIP5 erreichte Fortschritt allerdings nicht mehr so groß wie bei früheren Modellgenerationen.

Im deutschen Beitrag zu CMIP5 wurden Simulationen mit dem Erdsystemmodell MPI-ESM durchgeführt (Giorgetta et al.
2013). In Evaluationsstudien schneidet das MPI-ESM gegenüber anderen Modellen gut ab, z. B. bei der Simulation von Extremereignissen wie Hitzewellen oder Starkregen (Sillmann et al. 2013).

- Abb. 2.3 zeigt den Verlauf der mittleren globalen Oberflächentemperatur aus Simulationen und Beobachtungen seit Mitte des 19. Jahrhunderts. Die simulierte Temperatur einiger Modelle weicht deutlich von der beobachteten ab. Die meisten Modelle geben die beobachtete Variation der Oberflächentemperatur und besonders den langfristigen Anstieg recht gut wieder. Deutlich erkennbar sind die Auswirkungen großer Vulkanausbrüche. Zum Ende der Beobachtungszeit (2005) liegt die Änderung des Modellmittels etwa $0,15^{\circ} \mathrm{C}$ über der aus den Beobachtungen, was auf die deutliche Abschwächung der Erderwärmung in den vergangenen 15 Jahren zurückzuführen ist. Warum erwärmte sich die Erde in diesem Zeitraum langsamer? Gründe für diesen "Hiatus" werden auch im Fünften Sachstandsbericht diskutiert: Bei „mittlerem Vertrauen“ liegt das „grob zu gleichen Teilen“ an einem schwächeren Strahlungsantrieb und natürlichen Schwankungen (IPCC 2013a). Letztere ergeben sich wahrscheinlich aus einer veränderten vertikalen Wärmeverteilung im Ozean, sodass sich das Wasser an der Ozeanoberfläche - und damit auch die bodennahe Luft - weniger erwärmt hat als das Wasser in der Tiefe. Tatsächlich ist während der vergangenen 15 Jahre der Ozean insgesamt deutlich wärmer geworden und der Meeresspiegel angestiegen. Die Oberflächentemperatur hat sich jedoch nur geringfügig erhöht. Dass der Strahlungsantrieb nachgelassen 
hat, soll hauptsächlich zum einen an mehreren kleinen Vulkanausbrüchen liegen, die die Aerosolbelastung in der Stratosphäre (Atmosphärenschicht, die sich von etwa 15 bis $50 \mathrm{~km}$ Höhe erstreckt) erhöht haben, wodurch dort mehr Sonnenlicht zurückgestreut wird. Zum anderen ist die Sonne weniger aktiv als vor etwa 15 Jahren. Die Klimamodelle im CMIP5-Projekt simulieren in der Regel für die letzten 15 Jahre einen deutlich stärkeren Temperaturanstieg als beobachtet. Eine statistische Analyse von Modellen und Messdaten (Marotzke und Forster 2015) zeigt jedoch, dass man daraus nicht zwangsläufig auf Modellfehler schließen kann, sondern dass die Diskrepanz von der spontanen Variabilität des Klimas dominiert wird. Eine neue Analyse der globalen Mitteltemperatur (Karl et al. 2015) kommt zu dem Ergebnis, dass der Temperaturtrend der letzten 15 Jahre tatsächlich stärker ist als in bisherigen Analysen berechnet. Demnach wäre die Diskrepanz zwischen Modellen und Beobachtungen deutlich geringer als bisher angenommen. Das letzte Wort ist in der Diskussion um den „Hiatus" sicherlich noch nicht gesprochen.

Dank besserer Modelle und längerer Beobachtung lässt sich im Fünften noch klarer als im Vierten Sachstandsbericht nachweisen, dass der Mensch das Klima beeinflusst. So ist es jetzt „extrem wahrscheinlich", dass der Mensch mehr als die Hälfte des seit 1951 beobachteten Anstiegs der global gemittelten Temperatur verursacht hat. Ebenso gilt jetzt als ,sehr wahrscheinlich“, dass der Mensch mitverantwortlich ist für den seit 1979 beobachteten Rückgang des arktischen Meereises (IPCC 2013a).

Die CMIP5-Modelle werden nicht mehr nur für Projektionen über Zeitskalen von mehreren Jahrzehnten bis Jahrhunderten genutzt, sondern sie werden auch im Hinblick auf die Qualität sogenannter dekadischer Vorhersagen analysiert. Im aktuellen Sachstandsbericht (IPCC 2013a) wird abgeschätzt, dass bei Vorhersagen von 10 Jahren die Unsicherheit aufgrund interner Klimavariabilität deutlich höher ist als die Unsicherheit, die sich aus dem Emissionsverlauf ergibt. Umgekehrt ist deren Verhältnis, wenn man mehrere Jahrzehnte betrachtet. Die Vorhersagbarkeit relativ kurzer Zeiträume wurde im CMIP5-Projekt getestet: Vergangene Dekaden wurden mit beobachteten Anfangswerten simuliert und diese mit historischen Simulationen verglichen, die nicht mit Beobachtungen initialisiert wurden. Bezüglich der Oberflächentemperatur sowohl im globalen Mittel als auch in verschiedenen Regionen wie dem Nordatlantik, Teilen des Südpazifiks und dem tropischen Indischen Ozean wurden bessere Ergebnisse erzielt, in anderen Regionen dagegen schlechtere. Da die dekadische Vorhersage ein neues Forschungsgebiet ist, kann in den nächsten Jahren mit Verbesserungen gerechnet werden.

\subsubsection{Projektionen des zukünftigen Klimas}

Bei Projektionen des Klimas für das weitere 21. Jahrhundert schaut die Öffentlichkeit häufig auf die mittlere globale Oberflächentemperatur. - Abb. 2.4a zeigt ihren Anstieg nach den verschiedenen RCP-Szenarien bis Ende des 21. Jahrhunderts im Vergleich zum Mittel der Jahre 1986-2005. Im Szenario RCP8.5 steigt die Temperatur im CMIP5-Modellmittel um $3,7^{\circ} \mathrm{C}$. Wegen unterschiedlicher Ergebnisse der verschiedenen Modelle wird für den „wahrscheinlichen“ Temperaturanstieg ein Unsicherheitsbereich von $\pm 1,1^{\circ} \mathrm{C}$ um diesen Mittelwert herum angegeben (IPCC 2013a). Im Szenario RCP 2.6 steigt die Temperatur „wahrscheinlich“ nur um $1,0 \pm 0,7^{\circ} \mathrm{C}$. Szenario RCP2.6 geht von einer drastischen Verringerung der $\mathrm{CO}_{2}$-Emissionen bis hin zu sogenannten negativen Emissionen (z. B. durch Verbrennung von Biomasse und anschließender Abscheidung und Speicherung des emittierten Kohlendioxids) ab etwa 2070 aus. Nicht zu vergessen: Das öffentlich diskutierte Zwei-Grad-Ziel bezieht sich auf den mittleren Temperaturanstieg gegenüber der vorindustriellen Zeit. Seitdem ist die Temperatur bereits um etwa $0,8^{\circ} \mathrm{C}$ gestiegen. Auch ist die globale Erwärmung von den großen Ozeanflächen dominiert. Der Temperaturanstieg über dem Land liegt im Mittel jedoch „wahrscheinlich“ 1,4- bis 1,7-fach höher als der Anstieg über den Ozeanen (IPCC 2013a). Auch in Zukunft ist über den Kontinentalregionen ein stärkerer Anstieg als im globalen Mittel zu erwarten.

Die Erwärmung der Erdoberfläche bis zum Ende des 21. Jahrhunderts hängt nur geringfügig vom zeitlichen Verlauf der Emissionen ab. Wegen der langen Lebensdauer von Kohlendioxid und der Trägheit des Klimasystems schlagen hier vielmehr die über viele Jahrzehnte angehäuften Gesamtemissionen zu Buche (- Abb. 2.5). So wird die Zwei-Grad-Grenze voraussichtlich in etwa dann erreicht, wenn sich $800 \mathrm{Gt}$ (Mrd. Tonnen) Kohlenstoff aus anthropogenen $\mathrm{CO}_{2}$-Emissionen angesammelt haben. Je nach Emissionsszenario kann diese Marke früher oder später erreicht werden. Deutschland hat bisher etwa $23 \mathrm{Gt}$ Kohlenstoff (Boden et al. 2012) zu den Gesamtemissionen beigetragen.

Die historische Simulation des abnehmenden arktischen Meereises liegt in CMIP5 deutlich näher an den Beobachtungen als in der vorangegangenen CMIP-Phase - wenngleich die Modelle den Rückgang im Mittel immer noch unterschätzen. - Abb. 2.4b zeigt den projizierten zukünftigen Rückgang des arktischen Meereises. Demnach ist im RCP8.5-Szenario ab etwa 2050 die Arktis im Sommer fast komplett eisfrei. Auch für das antarktische Meereis wird ein Rückgang projiziert. Allerdings passen Modelle und Beobachtungen nicht zusammen: Während die Modelle im Mittel für die vergangenen 3 Jahrzehnte einen leichten Rückgang simulieren, hat sich das Eis aber tatsächlich leicht ausgedehnt.

Bis Ende des 21. Jahrhunderts (Mittel der Jahre 2081-2100) steigt den CMIP5-Modellen zufolge der Meeresspiegel global um $0,40 \pm 0,14 \mathrm{~m}$ (RCP2.6) bis $0,63 \pm 0,18 \mathrm{~m}$ (RCP8.5) verglichen mit der Zeit von 1986-2005. Etwas weniger als die Hälfte des Anstiegs geht auf das Konto der wärmebedingten Ausdehnung des Meerwassers. Außerdem schmelzen Gletscher sowie das grönländische und antarktische Inlandeis. Die Dynamik dieser Eismassen ist bisher in den CMIP5-Modellen meist nicht integriert, sondern wird offline berechnet. Ihr Beitrag zum zukünftigen Meeresspiegelanstieg gilt noch als sehr unsicher. Bekannt ist jedoch, dass der Meeresspiegel nicht überall gleich ansteigt. Das liegt daran, dass sich Bodendruck und Ozeandynamik regional unterschiedlich ändern. Auch wirken sich Änderungen der Eisbedeckung auf der Erdoberfläche auf das Gravitationsfeld der Erde nicht überall gleich aus. Das heißt: Der Meeresspiegel steigt also an einzelnen Küsten unterschiedlich stark.

Auch hinsichtlich der Entwicklung von Niederschlägen sagt der globale Wert wenig aus. In den Szenarien mit stärkeren Emissionen als im Szenario RCP2.6 liegt der Anstieg „sehr wahrscheinlich" bei 1-3\%, wenn sich die Erde um $1{ }^{\circ} \mathrm{C}$ erwärmt. Regional betrachtet heißt das: ,wet gets wetter, dry gets drier" (Held 
- Abb. 2.4 Änderung der oberflächennahen Lufttemperatur relativ zum Mittel der Jahre 1986-2005

(a). b Meereisbedeckung der Arktis im September in Mio. km² (als laufendes 5-Jahres-Mittel). Die Kurven zeigen die historische Simulation (schwarz) sowie die Szenarien RCP8.5 (rot) und RCP2.5 (blau). Rechts neben der Grafik sind Mittelwerte und Unsicherheiten der Jahre 2081-2100 für verschiedene Szenarien angegeben. Bei a zeigt die Linie das Multimodellmittel, die Schraffierung dessen Unsicherheit. Die Anzahl der verwendeten Modelle ist angegeben. Bei $\boldsymbol{b}$ ist das Multimodellmittel gepunktet. Die durchgezogenen Linien zeigen Mittelwerte jener fünf Modelle, die die beobachtete Meereisentwicklung am besten simulieren (RCP2.6 wurde nur von drei Modellen simuliert). Die Schattierung zeigt den Bereich zwischen Minimalund Maximalwert jener "besten" Modelle. (Modifizierte Abb. SPM.7, IPCC-AR5-WG1, IPCC 2013b)
- Abb. 2.5 Anstieg der mittleren globale Oberflächentemperatur (relativ zum Mittel der Jahre 18611880) als Funktion der kumulativen globalen $\mathrm{CO}_{2}$-Emissionen (in Mrd Tonnen Kohlenstoff) als Mittel aus verschiedenen CMIP5-Simulationen. Die Jahreszahlen stehen jeweils für das Mittel für die vorangehenden 10 Jahre und geben an, in welchem Jahrzehnt bei dem entsprechenden Szenario der jeweilige Wert erreicht wird. Die rote Fläche illustriert den Schwankungsbereich der Simulationen zu den unterschiedlichen RCP-Szenarien. Die schwarze dünne Linie und die graue Fläche stammen aus idealisierten CMIP5-Simulationen, bei denen die $\mathrm{CO}_{2}$-Konzentration jährlich um $1 \%$ erhöht wurde. Bei gleichen $\mathrm{CO}_{2}$-Emissionen werden hier niedrigere Temperaturen als in den RCP-Szenarien erreicht, weil in die RCPs Emissionen weitere Treibhausgase einfließen. (Modifizierte Abb. SPM.10, IPCC-AR5-WG1, IPCC 2013b)
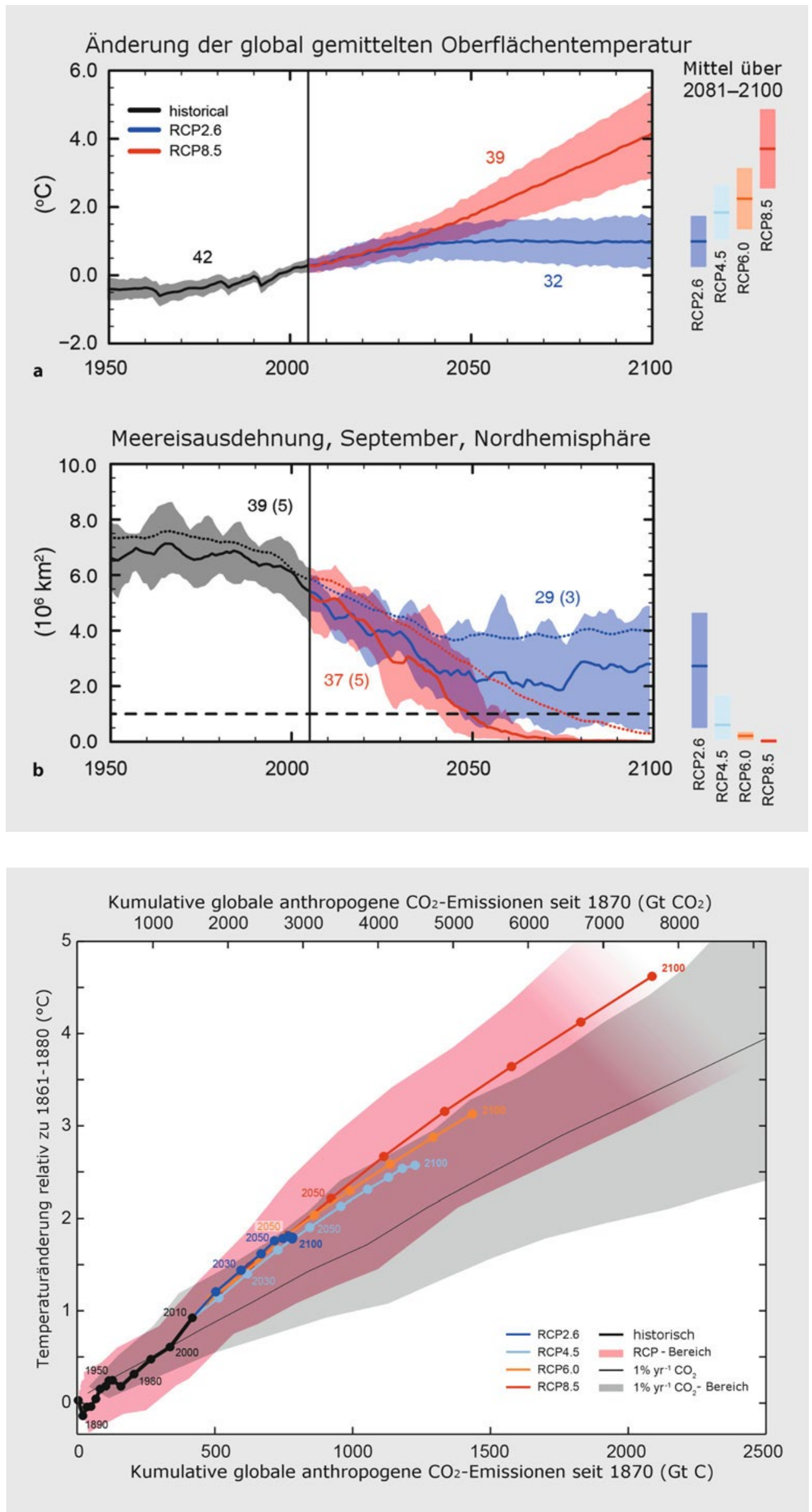
und Soden 2006). So auch in Europa: Während für den trockenen Mittelmeerraum weniger Niederschlag projiziert wird, soll es im nassen Skandinavien mehr regnen und schneien. Detailliertere Untersuchungen des regionalen Klimawandels werden mit regionalen Modellen durchgeführt ( Kap. 4), die Ergebnisse der globalen Modelle als Randbedingungen nutzen.

\subsection{Kurz gesagt}

Computermodelle des Klimas sind die einzig verfügbaren Werkzeuge für belastbare Klimaprojektionen. Diese beschreiben die Entwicklung des Klimas unter der Annahme eines Szenarios künftiger Emissionen von Treibhausgasen. Die im aktuellen Weltklimabericht benutzten Szenarien unterscheiden sich deutlich von früheren Szenarien. Insbesondere werden massive Maßnahmen zur Eindämmung des Klimawandels bis hin zu negativen $\mathrm{CO}_{2}$-Emissionen in der zweiten Hälfte des 21. Jahrhunderts berücksichtigt. Abhängig vom Szenario ergibt sich im Modellmittel ein mittlerer globaler Temperaturanstieg bis zum Ende des 21. Jahrhunderts um $1,7-4,4^{\circ} \mathrm{C}$, verglichen mit der Zeit von 1850 bis 1900. Über den Kontinenten wird sich die Atmosphäre deutlich stärker erwärmen als über den Ozeanen. Daneben sind weitere spürbare Veränderungen des Klimas zu erwarten: So wird z.B. der Meeresspiegel weiter ansteigen und das Meereis weiter zurückgehen.

\section{Literatur}

Arrhenius S (1896) On the influence of carbonic acid in the air upon the temperature of the ground. The London, Edinburgh and Dublin Philosophical Magazine and Journal of Science 5:237-276

Boden TA, Marland G, Andres RJ (2012) Global, regional and national fossil fuel $\mathrm{CO}_{2}$-emissions. Carbon Dioxide Information Analysis Center, Oak Ridge National Laboratory, US Department of Energy, Oak Ridge

Bony S, Stevens B, Held IH, Mitchell F, Dufresne J-L, Emanuel KA, Friedlingstein P, Griffies S, Senior C (2013) Carbon dioxide and climate: perspectives on a scientific assessment. In: Climate Science for Serving Society. Springer, Netherlands, S 391-413

Budyko MI (1969) The effect of solar radiation variations on the climate of the earth. Tellus 21(5):611-619

Charney JG, Arakaw A, Baker DJ et al (1979) Carbon Dioxide and Climate: a Scientific Assessment. National Academy of Sciences Press, Washington

Cubasch, U., D. Wuebbles, D. Chen, M.C. Facchini, D. Frame, N. Mahowald, and J.-G. Winther, 2013: Introduction. In: Climate Change 2013: The Physical Science Basis. Contribution of Working Group I to the Fifth Assessment Report of the Intergovernmental Panel on Climate Change [Stocker, T.F., D. Qin, G.-K. Plattner, M. Tignor, S.K. Allen, J. Boschung, A. Nauels, Y. Xia, V. Bex and P.M. Midgley (eds.)]. Cambridge University Press, Cambridge, United Kingdom and New York, NY, USA

Eyring V, Arblaster JM, Cionni I, Sedlacek J, Perlwitz J, Young PJ, Bekki S, Bergmann D, Cameron-Smith P, Collins WJ, Faluvegi G, Gottschaldt K-D, Horowitz LW, Kinnison DE, Lamarque J-F, Marsh DR, Saint-Martin D, Shindell DT, Sudo K, Szopa S, Watanabe S (2013) Long-term ozone changes and associated climate impacts in CMIP5 simulations. J Geophys Res Atmos 118. doi:10.1002/jgrd.50316

Flato G, Marotzke J, Abiodun B, Braconnot P, Chou SC, Collins W, Cox P, Driouech F, Emori S, Eyring V, Forest C, Gleckler P, Guilyardi E, Jakob C, Kattsov V, Reason C, Rummukainen M (2013) Evaluation of climate models. In: Stocker TF, Qin D, Plattner G-K, Tignor M, Allen SK, Boschung J, Nauels A, Xia Y, Bex V, Midgley PM (Hrsg) Climate Change 2013: The Physical Science
Basis. Contribution of working group I to the fifth assessment report of the Intergovernmental Panel on Climate Change. Cambridge University Press, Cambridge, United Kingdom and New York, NY, USA

Friedlingstein P, Meinshausen M, Arora VK, Jones CD, Anav A, Liddicoat SK, Knutti R (2014) Uncertainties in CMIP5 climate projections due to carbon cycle feedbacks. J Climate 27:511-526. doi:10.1175/JCLI-D-12-00579.1

Giorgetta MA, Jungclaus JH, Reick CH, Legutke S, Brovkin V, Crueger T, Esch M, Fieg K, Glushak K, Gayler V, Haak H, Hollweg H-D, Ilyina T, Kinne S, Kornblueh L, Matei D, Mauritsen T, Mikolajewicz U, Mueller WA, Notz D, Raddatz T, Rast S, Redler R, Roeckner E, Schmidt H, Schnur R, Segschneider J, Six K, Stockhause M, Wegner J, Widmann H, Wieners K-H, Claussen M, Marotzke J, Stevens B (2013) Climate and carbon cycle changes from 1850-2100 in MPI-ESM simulations for the coupled model intercomparison project phase 5. Journal of Advances in Modeling Earth Systems 5:572-597

Held IM, Soden BJ (2006) Robust responses of the hydrological cycle to global warming. J Climate 19:5686-5699

IPCC (2007) In: Solomon S, Qin D, Manning M, Chen Z, Marquis M, Averyt KB, Tignor M, Miller HL (Hrsg) eds. Cambridge University Press, Cambridge, United Kingdom and New York, NY, US

IPCC (2013a) In: Stocker TF, Qin D, Plattner G-K, Tignor M, Allen SK, Boschung J, Nauels A, Xia Y, Bex V, Midgley PM (Hrsg) Climate Change 2013: The physical science basis. Contribution of Working Group I to the Fifth Assessment Report of the Intergovernmental Panel on Climate Change. Cambridge University Press, Cambridge, United Kingdom and New York, NY, US

IPCC (2013b) Summary for policymakers. In: Stocker TF, Qin D, Plattner G-K, Tignor M, Allen SK, Boschung J, Nauels A, Xia Y, Bex V, Midgley PM (Hrsg) Climate Change 2013: The physical science basis. Contribution of Working Group I to the Fifth Assessment Report of the Intergovernmental Panel on Climate Change. Cambridge University Press, Cambridge, United Kingdom and New York, NY, USA

Karl TR, Arguez A, Huang B, Lawrimore JH, McMahon JR, Menne MJ, Peterson TC, Vose RS, Zhang H-M (2015) Possible artifacts of data biases in the recent global surface warming hiatus. Science 348(6242):1469-1472

Lacis AA, Schmidt GA, Rind D, Ruedy RA (2010) Atmospheric $\mathrm{CO}_{2}$ : Principal control knob governing earth's temperature. Science 330(6002):356-359

Lamarque J-F, Shindell DT, Josse B, Young PJ, Cionni I, Eyring V, Bergmann D, Cameron-Smith P, Collins WJ, Doherty R, Dalsoren S, Faluvegi G, Folberth G, Ghan DJ, Horowitz LW, Lee YH, MacKenzie IA, Nagashima T, Naik V, Plummer D, Righi M, Rumbold ST, Schulz M, Skeie RB, Stevenson DS, Strode S, Sudo K, Szopa S, Voulgarakis A, Zeng G (2013) The Atmospheric Chemistry and Climate Model Intercomparison Project (ACCMIP): overview and description of models, simulations and climate diagnostics. Geosci Model Dev 6:179-206. doi:10.5194/gmd-6-179-2013

Manabe S, Bryan K (1969) Climate calculations with a combined ocean-atmosphere model. Journal of the Atmospheric Sciences 26:786-789

Manabe S, Möller F (1961) On the radiative equilibrium and heat balance of the atmosphere. Monthly Weather Review 31:118-133

Manabe S, Wetherald RT (1967) Thermal equilibrium of the atmosphere with a given distribution of relative humidity. Journal of the Atmospheric Sciences 24:241-259

Marotzke J, Forster PM (2015) Forcing, feedback and internal variability in global temperature trends. Nature 517:565-570

Meinshausen M et al (2011) The RCP greenhouse gas concentrations and their extensions from 1765-2300. Climatic Change 109:213-241

Nakicenovic N, Swart R (2000) IPCC Special report on emissions scenarios. Cambridge University Press, Cambridge, UK, S 612

Otto A et al (2013) Energy budget constraints on climate response. Nature Geoscience 6:415-416. doi:10.1038/ngeo1836

Phillips NA (1956) The General Circulation of the Atmosphere: A Numerical Experiment. Quarterly Journal of the Royal Meteorological Society 82:123164

Sellers WD (1969) A global climatic model based on the energy balance of the earth-atmosphere system. Journal of Applied Meteorology 8(3):392-400

Sillmann J, Kharin VV, Zhang X, Zwiers F, Bronaugh D (2013) Climate extremes indices in the CMIP5 multimodel ensemble: Part 1. Model evaluation in the present climate. Journal of Geophysical Research: Atmospheres 118(4):1716-1733 
Taylor KE, Stouffer RJ, Meehl GA (2012) An Overview of CMIP5 and the experiment design. Bull Amer Meteor Soc 93:485-498. doi:10.1175/BAMSD-11-00094.1

Van Vuuren et al (2011) The representative concentration pathways: an overview. Climatic Change 109:5-31. doi:10.1007/s10584-011-0148-z

Open Access Dieses Kapitel wird unter der Creative Commons Namensnennung 4.0 International Lizenz (http://creativecommons.org/ licenses/by/4.0/deed.de) veröffentlicht, welche die Nutzung, Vervielfältigung, Bearbeitung, Verbreitung und Wiedergabe in jeglichem Medium und Format erlaubt, sofern Sie den/die ursprünglichen Autor(en) und die Quelle ordnungsgemäß nennen, einen Link zur Creative Commons Lizenz beifügen und angeben, ob Änderungen vorgenommen wurden.

Etwaige Abbildungen oder sonstiges Drittmaterial unterliegen ebenfalls der genannten Creative Commons Lizenz, sofern sich aus der Abbildungslegende oder der Quellreferenz nichts anderes ergibt. Sofern solches Drittmaterial nicht unter der genannten Creative Commons Lizenz steht, ist eine Vervielfältigung, Bearbeitung oder öffentliche Wiedergabe nur mit vorheriger Zustimmung des betreffenden Rechteinhabers oder auf der Grundlage einschlägiger gesetzlicher Erlaubnisvorschriften zulässig. 